\title{
Using Lagrangean Relaxation for Service Location Planning with QoS constraints in Large-Scale Networks
}

\author{
Zille Huma Kamal, Ala Al-Fuqaha, and Ajay Gupta
}

\begin{abstract}
Current trends in computing indicate that there is a great potential for service oriented computing and similar technologies, such as Cisco's Application Oriented Networks, where services provide a higher-level of abstraction to traditional applications. In such cases, providing and consuming services and establishing a relationship between consumers (users of services) and producers (providers of services) are still challenging and vastly researched aspects. We define a Service Location Planning Problem (SLP) that is novel in the approach it takes to match consumers to producers, such that the cost of providing services is minimized while taking into account quality of service constraints of throughput and delay. In previous work, we presented an Integer Linear Programming (ILP) model to formulate the service location planning problem. In this paper, we present Lagrangean Relaxation of the model for large-scale networks. We use the approach of Trick [3] to decrease computational overheads.
\end{abstract}

Index Terms-Lagrangean Relaxation, Service Location Planning Problem, Application Oriented Networks, Opportunistic Networks

\section{INTRODUCTION}

$I_{c}$ N ANY COMPUTING PARADIGM, which abstracts traditional computer applications to the level of services, the challenge lies in advertising these services and optimally matching consumers requesters to providers of such services. Numerous service discovery protocols, e.g. Jini, Salutation, etc. and standards, such as XML, WSDL, OWL, etc. are in existence to achieve such service advertisement and consumption goals.

Cisco Systems, takes this form of service oriented computing to a higher-level with their new technology of Application Oriented Networks (AON), which relocates application services from end nodes (end-points) in the network to the routers and appliances in the network [4]. This is a huge impact technology since it allows application-level message intelligence at the router level.

Manuscript received September 28, 2007.

Z. H. Kamal, Student IEEE Member, is with the Department of Computer Science, Western Michigan University, Kalamazoo, MI 49008 USA (phone: 269-267-5070; fax: 269-276-3122; e-mail: zkamal@cs.wmich.edu).

A. Al-Fuqaha, IEEE Member, is with the Department of Computer Science, Western Michigan University, Kalamazoo, MI 49008 USA (e-mail: alfuqaha@cs.wmich.edu).

A. Gupta, IEEE Senior Member, is with the Department of Computer Science, Western Michigan University, Kalamazoo, MI 49008 USA (e-mail: gupta@cs.wmich.edu).
This approach has two key benefits: First, AON routers route application-layer messages, such as stock quotes, weather and news alerts, etc., to the appropriate application service rather than an arbitrary IP address. Second, services can be installed on AON routers. This enables faster satisfaction of service demand requests, as these requests are not propagated to the end-points (outside) of the network, rather interpreted and satisfied within the network. Other technologies such as Opportunistic Networks (Oppnets) [10] and Mobile Computing can also be viewed in terms of the service oriented paradigm discussed.

In the Service Location Planning (SLP) Problem, we have a network of nodes and a set of services that are being requested by nodes in the network. The problem associates a service installation cost with every service that is to be installed in the network to meet the requirements. These installation costs vary from one node to another. The goal is to minimize the service installation cost, promote service federation and furthermore meet quality of service (QoS) requirements of throughput and delay. This is a novel approach, since to the best of our knowledge, service location-planning and QoS requirements have not been previously factored into AON and service discovery protocols and standards.

The SLP problem can seem similar to the popular Uncapacitated/Capacitated Facility Location Problems (UCFL) [5], however, a closer look at the SLP and UCFL will show that the SLP is broader and more complex than the NPhard UCFL [6]. A literature review in our previous work [9] demonstrates the flexibility of our SLP formulation.

In this paper, our contribution is use of the Lagrangean Relaxation approximation approach to perform near optimal service location planning in large-scale networks in terms of the number of nodes and offered and requested services.

The rest of the paper is organized as follows. Section II gives a review of various Lagrangean Relaxation techniques. Section III briefly discusses the ILP model used to formulate the SLP problem and Section IV presents the Lagrangean Relaxation of the SLP problem for large-scale networks. Section V discusses scenarios and compares Lagrange Relaxation and ILP results. We summarize and conclude our findings and contributions in section VI.

\section{BACKGROUND - APPROXIMATION TECHNIQUES}

Mathematical formulation of research problems as 
optimization problems is a research methodology that is used in diverse areas of research and study ranging from economics to physics to computer science. When a research problem can be successfully formulated as a mathematical problem/model, it can be expressed and defined unambiguously and solved accurately. However, often hard or complex formulations cannot be solved effectively or are computationally intensive. Furthermore, some mathematical formulations can only be solved on a small-scale and when large-scale scenarios are considered, the problem becomes too large or complex to be solved using traditional ILP/LP solve engines (e.g. CPLEX, LpSolve [8], etc.). In such cases, approximation techniques, such as Linear Programming relaxation and Lagrangean Relaxation (LR), are used to solve the problem.

We are interested in Lagrangean Relaxation of ILP problems, where multipliers are attached to the complicating constraints and moved into the objective function [2]. This presents a dual of the original problem that is often easier to solve and known as the Lagrangean dual [2] and the multipliers of the complicating constraints are termed as Lagrangean multipliers.

The multipliers are used to control the Lagrangean dual, such that, when a solution to the problem violates the complicating constraint, the multipliers are used to penalize the objective function. The optimal value of the Lagrangean dual objective function gives a lower bound for minimization problems and an upper bound for maximization problems, if multipliers are always positive.

Let's briefly illustrate the Lagrangean Relaxation technique. Consider for example, an ILP minimization problem $L$ defined as follows.

$$
\begin{array}{ll}
L=\min & f x \\
\text { subject to: } & A x \leq b \\
& C x=d \\
& x=\{0,1\}
\end{array}
$$

Where, $x$ is the problem variable and $f, A$ and $C$ are problem dependent input parameters and $b$ and $d$ are constants. Note, that the third constraint makes this a combinatorial problem.

Let us assume that the first constraint is the complicating constraint. Using the Lagrangean Relaxation for ILP problems, the Lagrangean dual $L^{*}$ can be formulated as follows.

$$
\begin{array}{ll}
\qquad L^{*}=\min ( & \left.f x+\lambda_{1}(A x-b)\right) \\
\text { subject to: } \quad & C x=d \\
& x=\{0,1\}
\end{array}
$$

Where, $\lambda_{1}$ is the Lagrangean multiplier.

$\therefore$ for a valid solution for $x$ and for $\lambda_{1} \geq 0$,

$\Rightarrow A x \leq b \Rightarrow A x-b \leq 0$

$\Rightarrow L^{*} \leq L \quad$ (note: $L^{*}=L$ is not guaranteed)

This is how, the Lagrangean multipliers are used to control the solution $x$ and penalize the objective function when a solution to $x$ violates the complicating constraint(s).

Often the aim of Lagrangean relaxation is to show that a bound exists. However, our goal is to extend this idea to devise approximate solutions. Therefore, in our algorithm we remove the restriction that the Lagrange multipliers have to be positive.

Further the goal of the Lagrange relaxation technique is to iteratively update the Lagrange multipliers through different algorithms, such as subgradient, surrogate gradient [1], [2], a technique we refer to as the CMU (Carnegie Mellon University) technique [3], etc. Irrespective of the technique used, the multipliers are updated such that they represent the cost of violating the constraint it represents. We next briefly review the subgradient, surrogate gradient and the CMU techniques.

\section{A. Subgradient and Surrogate Gradient Techniques}

For simplicity we omit the mathematical proofs and theorem and just discuss the intuition behind subgradient and surrogate gradient methods and refer readers to [1] for a more detailed discussion.

In the subgradient method, the multipliers are updated according to the step-size and subgradient of the dual Lagrangean function at each iteration. The step-size is an application dependent variable and can be computed according to a mathematical formula or an easier rule that has empirically performed well is to set step size to 2 and halved whenever Lagrangean dual bound fails to increase after some fixed number of iterations [2]. Computing the subgradient requires a solution of all the sub-problems [1].

The surrogate gradient minimizes time by eliminating the need for a solution to all sub-problems, as in subgradient approach [2].

\section{B. CMU Technique}

In the multiplier update method of [3], which we termed CMU method, computation time is shortened significantly as the need to compute any subgradient or surrogate gradients is eliminated and a very straight forward approach is used to update the multipliers. We adapted this algorithm for minimization problems and present it in Fig. 1. In short, the multipliers are updated by a constant $k$, in each iteration based on whether they violate or give slack to a constraint.

Two properties are important in evaluating which relaxation technique to use, the sharpness of the bounds produced and the amount of computation time required to obtain these bounds

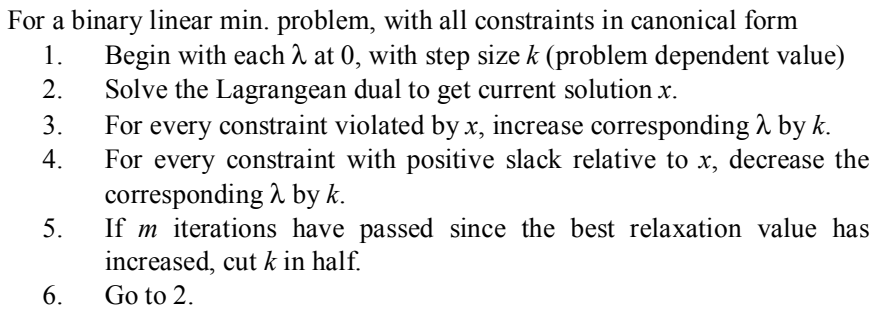

Fig. 1. CMU Lagrange Relaxation Technique [3] adapted for binary linear minimization problems.

[2]. Usually, there is a tradeoff between these two properties [2].

Our goal was simplicity, in terms of computationally intensity, thus we adapted the CMU Lagrangean Relaxation technique to extend the SLP model for large-scale networks. 


\section{INTEGER LiNEAR MODEL FOR SERVICE LOCATION PLANNING PROBLEM (SLPP)}

In this section we present the formal definition of the SLP and its formulation as an ILP problem in brevity.

\section{A. Formal Definition}

The Service Location Planning (SLP) problem can be defined as follows.

Given: A network graph $\mathrm{G}=(V, E)$ and a set of services $S$, where $V$ is set of vertices/nodes and $E$ is the set of edges. There exists a set of consumers, $V^{C} \subseteq V$, such that nodes $v \in V^{C}$ are requesting a service(s), $s_{i} \in S, i=1 \ldots|S|$ and have a throughput and delay demand associated with each request. There is also a service installation cost associated with each service $s$ on a node $n$ in the network $\mathrm{G}$ and a service discount $d$ is given to promote service federation (multiple service installations on the same node).

The cost of installing a service $s$ on a node $n$ may depend on: (i) cost of accessing the node, i.e. either physically/manually or in terms of number of hops, (ii) storage capacity of the node, (iii) processing capacity of the node, (iv) installing the application (i.e. the service $s$ ) itself, etc.

Installing multiple services on a node is promoted because based on the service installation costs, it will be beneficial to a producer $p$, if its service $s 1$ is installed on node $n$ at the same time when another producer $q$ 's, service $s 2$ is being installed on $n$. This way, at the least the cost of accessing $n$ is shared between the two producers. Resource reallocation at node $n$ can be optimized taking demands of s1 and s2 into account. These factors can be formulated as a discount in the service installation cost.

Problem: Install services on a set of producers $V^{P} \subseteq V$, such that the service installation cost incurred is minimal and all throughput and delay requirements are satisfied, while also satisfying the underlying link layer capacities (Note: producers can also be consumers of services).

\section{B. Input}

The inputs of the problem can be delineated as:

1. $n=$ number of nodes in the network, i.e. $|\mathbf{V}|$.

2. $s=$ maximum number of services requested.

3. $p=$ maximum number of useful paths in the network.

4. $e=$ total number of links/edges in the network, i.e. $|\mathbf{E}|$.

5. Path-link matrix $\mathbf{L}$ is a binary $p \times e$ dimensional that indicates whether a link is used in a path or not.

6. Bandwidth vector $\mathbf{B}$ is e dimensional that gives capacity of a link/edge and $b_{\max }$ is maximum link capacity.

7. Service installation cost matrix $\mathbf{C}$, is $n \times s$ dimensional that quantifies the cost for installing a service on a node.

8. Discount $\Gamma$ is given for multiple services installations on the same node.
9. A three dimensional binary routing matrix, $\mathbf{R}$ that indicates the path used between a source-destination pair in the network.

10. A throughput demand matrix $\mathbf{T}$ that gives the throughput required for a service at a consumer.

11. A delay demand matrix $\mathbf{D}$ that is the maximum delay allowed for a service at a consumer.

12. A load-delay lookup table $\mathbf{Q}$ that approximates the delay, due to queuing, transmission and propagation, on a link given the load on the link, $q_{\max }$ is maximum load and $q$ is the interval of load values in $\mathbf{Q}$.

\section{Variable}

The variables of the problem can be defined as follows:

1. A binary service location matrix $\mathbf{X}$ is $n \times s$ dimensional, that indicates whether a service is installed on a node or not.

2. A path-service capacity matrix $\mathbf{Z}$ is $p \times s$ dimensional, that quantifies the capacity of a service on a path.

3. An $n$ dimensional service installation indicator vector $U$ that indicates whether multiple services are installed on a node.

4. A binary service-path indicator (normalized $\mathbf{Z}$ ) matrix $\mathbf{Y}$ is $p \times s$, and indicates if a service uses a path or not.

5. An $e$ dimensional link load vector $\mathbf{V}$ that quantifies the load on a link.

6. An $e$ dimensional link-delay vector $\mathbf{G}$ that gives the delay on a link.

7. A $p$ dimensional path delay vector $\mathbf{H}$, gives the total end-to-end delay on a path

Variable $\mathbf{X}$ is also the output of the problem since it gives the optimal service installation configuration.

\section{Formulation}

Based on these inputs and variables the ILP model is formulated to minimize the cost of service installation.

$$
\min \left\{\sum_{i=1}^{n} \sum_{k=1}^{s} C_{i, k} \cdot X_{i, k}+\sum_{i=1}^{n} \Gamma \cdot U_{i}\right\}
$$

\section{subject to:}

(1) To enable service federation, a node that provides multiple services has its binary service installation indicator set to 1 .

$\sum_{k=1}^{s}\left(X_{i, k}\right)-p \cdot b_{\max } \cdot U_{i} \leq 0,-\sum_{k=1}^{s}\left(X_{i, k}\right)+U_{i} \leq 0, \forall 1 \leq i \leq n$

(2) To meet throughput requirements, the sum of all service capacities being provided via all paths to a consumer must meet the required throughput of the consumer for that service

$-\sum_{i=1}^{n} \sum_{m=1}^{p} R_{i, j, m} \cdot Z_{m, k} \leq-T_{j, k}, X_{i, k}-\sum_{j=1}^{n} \sum_{m=1}^{p} R_{i, j, m} \cdot Z_{m, k} \leq 0$,

$-p \cdot b_{\max } \cdot X_{i, k}+\sum_{j=1}^{n} \sum_{m=1}^{p} R_{i, j, m} \cdot Z_{m, k} \leq 0, \quad \forall 1 \leq i \leq n, 1 \leq k \leq s$ 
(3) To meet network link layer constraints, the sum of all service capacities that traverse a link must be less than the bandwidth of the link.

$$
\sum_{m=1}^{p} \sum_{k=1}^{s} L_{m, l} \cdot Z_{m, k} \leq B_{l}, \quad \forall 1 \leq l \leq e
$$

(4) Binary variable $\mathrm{Y}$ is a normalized $\mathrm{Z}$ value.

$-p \cdot b_{\max } \cdot Y_{m, k}+Z_{m, k} \leq 0, Y_{m, k}-Z_{m, k} \leq 0$,

$$
\forall 1 \leq m \leq p, 1 \leq k \leq s
$$

(5) The load on a link is computed as sum of all service capacities on all paths that traverse that link.

$$
\begin{aligned}
& V_{l}-\sum_{m=1}^{p} \sum_{k=1}^{s}\left(L_{m, l} \cdot Z_{m, k}\right) \leq 0,-V_{l}+\sum_{m=1}^{p} \sum_{k=1}^{s}\left(L_{m, l} \cdot Z_{m, k}\right) \leq 0, \\
& \forall 1 \leq l \leq e
\end{aligned}
$$

(6) The load on the link is rounded up to the nearest $q^{\text {th }}$ multiple.

$$
V_{l}-q \cdot a_{l} \leq 0, \quad-V_{l}+q \cdot a_{l} \leq 0, \quad \forall 1 \leq l \leq e
$$

(7) Binary free variables $c$ and $k$ are used to lookup the load and delay value in the load-delay lookup table, respectively.

$V_{l}-q \cdot i-\left(b_{\max }+q_{\max }\right) \cdot c_{l, i} \leq 0,-V_{l}+q \cdot i-\left(b_{\max }+q_{\max }\right) \cdot c_{l, i} \leq 0$,

$\sum_{i=1}^{\left|b_{l}\right|} c_{l, i} \leq \frac{b_{l}}{q}, k_{l, i}+c_{l, i} \leq 1,-k_{l, i}-c_{l, i} \leq 1, \quad \forall 1 \leq l \leq e, 1 \leq i \leq \frac{b_{l}}{q}+1$

(8) The delay on a link is simply computed as the load-delay lookup value.

$G_{l}-\sum_{i=1}^{|Q|}\left(k_{l, i} \cdot Q_{i}\right) \leq 0, \quad-G_{l}+\sum_{i=1}^{|Q|}\left(k_{l, i} \cdot Q_{i}\right) \leq 0, \quad \forall 1 \leq l \leq e$

(9) The delay on a path is computed as the sum of the delay across all links in the path.

$$
H_{m}-\sum_{l=1}^{e}\left(L_{m, l} \cdot G_{l}\right) \leq 0, \quad-H_{m}+\sum_{l=1}^{e}\left(L_{m, l} \cdot G_{l}\right) \leq 0, \quad \forall 1 \leq m \leq p
$$

(10) The delay on all paths providing a service $k$ to a consumer $j$ must not exceed delay constraint $D_{j, k}$.

$$
\begin{array}{ll}
-R_{i, j, m} \cdot Y_{m, k}-r_{i, j, k, m} \leq-1, & R_{i, j, m} \cdot Y_{m, k}+r_{i, j, k, m} \leq 1, \\
H_{m}-q_{\max } \cdot r_{i, j, k, m} \leq D_{j, k}, & \forall 1 \leq i, j \leq n, 1 \leq k \leq s, 1 \leq m \leq p
\end{array}
$$

(11) Only paths between a consumer and provider should have service capacities other unused paths should not have any service capacities.

$$
\begin{array}{r}
-q_{\max } \cdot p \sum_{i=1}^{n} \sum_{m=1}^{p} R_{i, j}^{m} Y_{m, k} \leq-T_{j, k}, \quad \sum_{i=1}^{n} \sum_{m=1}^{p} R_{i, j}^{m} Y_{m, k} \leq T_{j, k}, \\
\forall 1 \leq j \leq n, 1 \leq k \leq s
\end{array}
$$

\section{LAGRANGeAn RelaXation OF SERVICE LOCATION PLANNING PROBLEM}

In our previous work [9], we solved the ILP formulation presented in section III using the LpSolve [8] engine. In this paper we present the Lagrangean relaxation to extend our results to large-scale networks. The use of relaxation techniques, approximation techniques and other heuristics and meta-heuristics may yield suboptimal results, but allowing solution for large-scale networks that cannot be handled by LP solvers.

We discussed the simple CMU technique for Lagrange relaxation in section II and in Fig. 1 presented an adaptation of the CMU technique for binary linear minimization problems.

A scrutiny of the CMU technique indicates its applicability to binary linear problems. Therefore, we extended the CMU technique for integer linear problems by only relaxing those constraints that consist of binary variables such as the constraints in group (1), and those constraints pertaining to the computation of variable $k$ in group (7) in the ILP formulation of section III.

For the service location planning problem discussed in this paper, there are various levels of complexity. The dimensions of complexity in a SLP can be delineated in terms of number of nodes, $n$, services, $s$, paths, $p$, links/edges, $e$, bandwidth capacity, $b_{l}$, and lookup table interval, $q$.

Increasing any of these dimensions causes the problem to grow tremendously. For example, consider a problem with 6 nodes, 2 services and 22 paths, the number of variables in this problem is 480 , where as, a 6 node, 3 service and 22 path problem has 538 variables, leaving all other dimensions to be the same across the two problems, this is an increase of 58 variables just with respect to an increase of one service in the SLP problem.

Therefore, we can form different scenarios even with the same number of nodes in a network, by varying any of the other dimensions of the problem, e.g. the number of services and, or paths and, or bandwidth capacities.

\section{Test Scenarios And Results}

In this section we compare results of Lagrangean relaxation for small and larger-scale scenarios.

We consider a small network of 6 nodes and a large scale network of 24 nodes. The topology used is illustrated in Fig. 2. It illustrates a carrier's nation-wide ISP backbone network technology [7] with 24 nodes and we abstract smaller network topologies from this larger topology, e.g. the 6 node network topology is formed by nodes $1,2,3,4,6,7$.

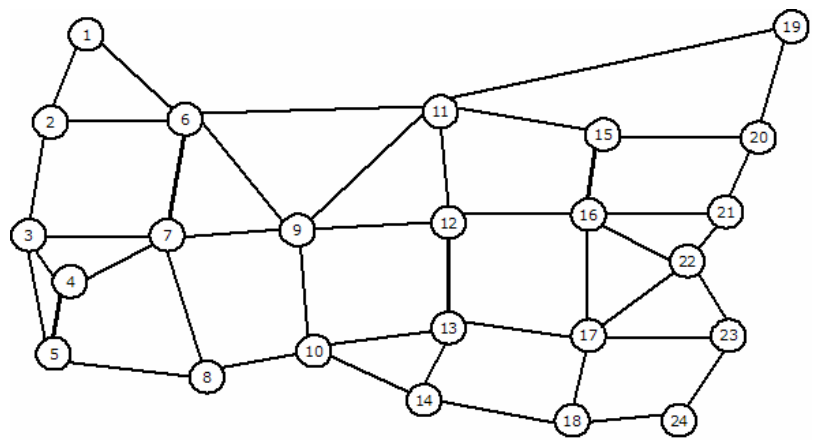

Fig. 2. A carrier's nation-wide IP backbone network topology [7]

We compare the reduction in the number of constraints in a problem in Fig. 3 (also tabulated in Table 1). The figure illustrates a reduction of $16-18 \%$ of the total constraints, i.e 
an average improvement of $17.42 \%$ (c.f. Table 1). This yields reductions of upto 729 constraints in a 24 node, 2 service and 58 path network setup. We reduce the complexity of the problem by only utilizing useful alternate paths (with a minimum number of edges traversed), rather than all possible paths between all source and destination pairs, which in itself is a hard problem.

In Fig. 3, the scenarios are delineated as $x \mathrm{n}-y \mathrm{~s}-z \mathrm{p}$, where $x, y$ and $z$ are the number of nodes, services and paths respectively. If two scenarios are labeled the same, then the difference is the bandwidth capacity of an underlying link(s). We keep the number of edges (e) constant across scenarios with same $n, s$, $p$ dimensions. Also, same lookup table is used in all scenarios.

Table 1 shows how Lagrangean relaxation bounds the ILP objective function value. It should be noted that the Lagrangean dual does not always represent a lower bound on the optimal solution (ILP cost) for all scenarios since we allowed multipliers to be negative.

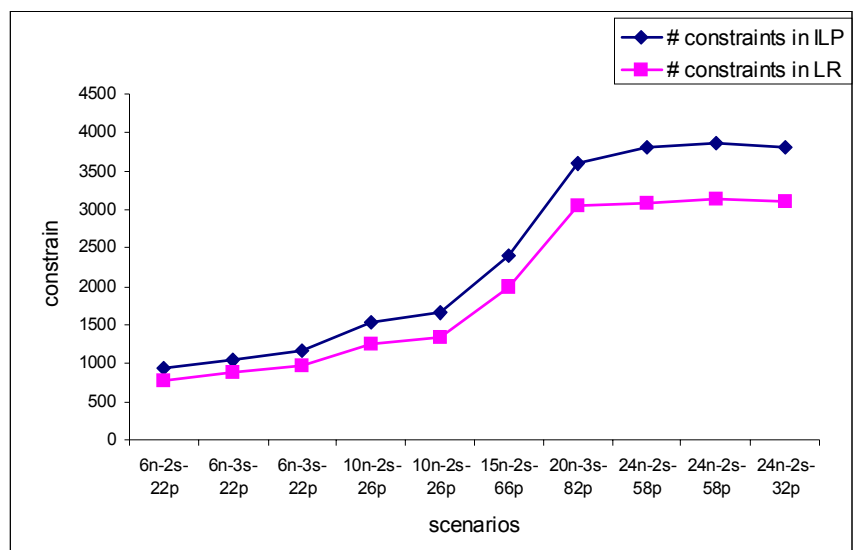

Fig. 3. Reduction in constraints with Lagrange relaxation versus ILP.

\section{CONCLUSION}

In conclusion, we illustrated a novel service location planning problem that is unique in the way it relates consumers to producers while taking into account individual throughput and delay requirements and not exceeding underlying link layer capacities.

Our contribution lies in the adaptation and implementation of a simple technique for updating Lagrange multipliers and approximating the optimal service location planning problem for large-scale networks.

Our results demonstrate an improvement of $17 \%$ in the number of constraints, on average, when using the Lagrange relaxation technique for the service location planning problem. Currently, this shows a significant improvement, however, since no prior work has been done in this area, there is no benchmark against which this improvement can be compared.

Future work includes further reducing the number of constraints by re-modeling the service location planning problem using only binary variables, developing a simulation model that be used to compare these Lagrange Relaxation results, and comparing results from different Lagrangean
TABLE I

ILP COST AND LR LOWER BOUND

\begin{tabular}{|c||c|c||c|c||c||}
\hline Scenario & $\begin{array}{c}\text { ILP } \\
\text { cost }\end{array}$ & $\begin{array}{c}\text { LR } \\
\text { lower } \\
\text { bound }\end{array}$ & $\begin{array}{c}\# \\
\text { constr. } \\
\text { in ILP }\end{array}$ & $\begin{array}{c}\# \\
\text { constr } \\
\text { in LR }\end{array}$ & $\begin{array}{c}\text { \% } \\
\text { improvement } \\
\text { in \# constr. }\end{array}$ \\
\hline $6 n-2 s-22 p$ & 240 & 219.62 & 940 & 778 & 17.23 \\
\hline $6 n-3 s-22 p$ & 340 & 324.93 & 1038 & 876 & 15.61 \\
\hline $6 n-3 s-22 p$ & 340 & 298 & 1158 & 966 & 16.58 \\
\hline $10 n-2 s-26 p$ & 240 & 220 & 1528 & 1250 & 18.19 \\
\hline $10 n-2 s-26 p$ & 180 & 168 & 1656 & 1346 & 18.72 \\
\hline $15 n-2 s-66 p$ & 60 & 48 & 2395 & 2001 & 16.45 \\
\hline $20 n-3 s-82 p$ & 100 & 68 & 3594 & 3050 & 15.14 \\
\hline $24 n-2 s-58 p$ & 160 & 236 & 3810 & 3095 & 18.77 \\
\hline $24 n-2 s-58 p$ & 280 & 320 & 3872 & 3143 & 18.83 \\
\hline $24 n-2 s-32 p$ & 160 & 220 & 3819 & 3104 & 18.72 \\
\hline
\end{tabular}

Average improvement $\mathbf{=} \mathbf{1 7 . 4 2}$

\# constr. $=$ number of constraints

$\mathrm{LR}=$ Lagrangean Relaxation

relaxation techniques in terms of accuracy, complexity in implementation and execution time.

\section{ACKNOWLEDGMENT}

The authors would like to acknowledge Computational Science Center and Information Technology and Image Analysis (ITIA) Center. Any opinions, finding, conclusions or recommendation expressed in the paper are those of the authors and do not necessarily reflect the views of the funding agencies or institutions.

\section{REFERENCES}

X. Zhao, P.B. Luh, and J. Wang, "Surrogate Gradient Algorithm for Lagrangian Relaxation," Journalrnl. of Optimization Theory and Applications, 100 (3), March 1999, pp. 699 - 712.

[2] M. L. Fisher, "The Lagrangian Relaxation Method For Solving Integer Programming Problems," Management Sci., 27(1), 1981. Michael A. Trick, "An application oriented tutorial on relaxations," Carnegie Mellon University, Pittsburgh, February, 1996. Last accessed online: http://mat.gsia.cmu.edu/mstc/relax/relax.html

[4] Cisco Systems Inc. 2006. "Introducing Cisco Application-Oriented Networking-A CIO Brief," Online, httpe://www.cisco.com

[5] D. B. Shmoys, C. Swamy, and R. Levi, "Facility Location with Service Installation Costs," Proc.eedings of the fifteenth15th annual ACM-SIAM symp.osium on Discrete algorithms, 2004

[6] M. G. C. Resende and R. F. Werneck, "A Hybrid Multistart Heuristic for the Uncapacitated Facility Location Problem," AT\&T Labs Research Technical Report (September 15), NJ, 2003.

[7] K. Zhu, available online, last accessed September 9, 2007, http://networks.cs.ucdavis.edu/ zhuk/toplogies.html

[8] M. Berkelaar, K. Eikland and P. Notebaert, "lp solve 5.5.0.10," Online, last accessed: http://psolve.sourceforge.net/5.5/

[9] Z. H. Kamal, A. Al-Fuqaha, and A. Gupta, "A service location problem with QoS constraints," Proceedings of 2007 International Conference on Wireless Communications and Mobile Computing (IWCMC'07), Hawaii, USA, 2007, pp. 641-646.

[10] Lilien, L., Kamal, Z. H., Bhuse, V., and Gupta, A. Opportunistic Networks: The Concept and Research Challenges in Privacy and Security. Book chapter in: Mobile and Wireless Network Security and Privacy ed. by K. Makki et al., Springer Science+Business Media, Norwell, Massachusetts, 2007. 